\title{
Hydrogenation of GaSb/GaAs quantum rings
}

\author{
P. D. Hodgson, ${ }^{1, a)}$ M. Hayne, ${ }^{1}$ M. Ahmad Kamarudin, ${ }^{1,2}$ Q. D. Zhuang, ${ }^{1}$ S. Birindelli, ${ }^{3}$ \\ and M. Capizzi ${ }^{3}$ \\ ${ }^{1}$ Department of Physics, Lancaster University, Lancaster LAI 4YB, United Kingdom \\ ${ }^{2}$ Department of Physics, Universiti Putra Malaysia, 43400 UPM Serdang, Selangor, Darul Ehsan, Malaysia \\ ${ }^{3}$ Dipartimento di Fisica, Sapienza Universita ‘ di Roma, Piazzale A. Moro 2, 00185 Roma, Italy
}

(Received 22 July 2014; accepted 20 August 2014; published online 28 August 2014)

\begin{abstract}
We present the results of photoluminescence measurements on hydrogenated type-II GaSb/GaAs quantum dot/ring $(\mathrm{QD} / \mathrm{QR})$ samples at temperatures ranging from $4.2 \mathrm{~K}$ to $400 \mathrm{~K}$. Hydrogenation is found to suppress optically induced charge depletion (associated with the presence of carbon acceptors in this system). A redshift of the QD\QR emission energy of a few tens of meV is observed at temperatures $\geq 300 \mathrm{~K}$, consistent with a reduction in average occupancy by $\sim 1$ hole. These effects are accompanied by a reduction in PL intensity post-hydrogenation. We conclude that although hydrogenation may have neutralized the carbon acceptors, multiple hole occupancy of type-II $\mathrm{GaSb} / \mathrm{GaAs} \mathrm{QD} / \mathrm{QRs}$ is very likely a precondition for intense emission, which would make extending the wavelength significantly beyond $1300 \mathrm{~nm}$ at room temperature difficult. (C) 2014 AIP Publishing LLC. [http://dx.doi.org/10.1063/1.4894413]
\end{abstract}

Self-assembled quantum dots (QDs) are the subject of intense research because of their interesting physical properties and possibilities for device fabrication in a range of electronic and optoelectronic applications. ${ }^{1-3}$ Whilst type-I QDs have been investigated extensively, their type-II counterparts have received less attention. However, the properties of type-II QDs have stimulated increasing interest in recent years. ${ }^{4-7}$ Type-II GaSb/GaAs nanostructures provide a very deep hole confinement potential, spatially separating them from the unconfined electrons in the surrounding GaAs matrix. Therefore, the binding energy is significantly smaller than in the type-I system but, nevertheless, due to the lack of electron confinement, the Coulomb interaction dominates the physics. ${ }^{5,8}$ The spatial separation of carrier species makes $\mathrm{GaSb} / \mathrm{GaAs} \mathrm{QD} / \mathrm{quantum}$ rings (QRs) candidates for use in solar cells where fast electron hole separation is desirable. ${ }^{4,9}$ In addition, the large confinement energies, negligible charging barriers, and strong 3-dimensional hole confinement in $\mathrm{GaSb} / \mathrm{GaAs} \mathrm{QD} / \mathrm{QRs}$ make them promising for use in memory devices. ${ }^{10,11}$ The deep hole confining potential also raises the possibility of GaAs-based lasers operating at telecommunications wavelengths. ${ }^{12-14}$ Deep-level-transientspectroscopy experiments on $\mathrm{GaSb} / \mathrm{GaAs}$ QD samples grown by different groups have measured hole ground-state localisation energies of 460,610 , and $760 \mathrm{meV} .^{15,16}$ Hence, we might expect that $300 \mathrm{~K}$ emission from $\mathrm{GaSb} / \mathrm{GaAs} \mathrm{QD} / \mathrm{QRs}$ should be $\sim 0.61 \mathrm{eV}$ below the GaAs band-gap, i.e., at $1.42-0.61=0.81 \mathrm{eV}(1530 \mathrm{~nm})$. In practice, the longest room-temperature emission peak reported to date is $1332 \mathrm{~nm}$ $(0.93 \mathrm{eV}) .{ }^{14}$ The blueshift in emission with increasing excitation power that is a feature of type-II systems, ${ }^{12,14,17-20}$ is clearly a limiting factor in reaching longer wavelengths. There are believed to be two mechanisms which cause this behaviour in $\mathrm{GaSb} / \mathrm{GaAs} \mathrm{QD} / \mathrm{QRs}$ : capacitive charging,

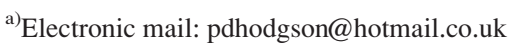

which is the dominant effect, and band bending, which is much weaker. ${ }^{21}$

In a few cases, redshifts of the emission energy with increasing excitation power (which precedes the blueshift) have also been observed in these nanostructures. ${ }^{22,23}$ These redshifts are the result of optically induced charge depletion (OICD). ${ }^{24}$ OICD is a dynamic process involving interactions between photogenerated carriers, a nanostructure and donor or acceptor states in the surrounding semiconductor material (Fig. 1). In short, OICD is the optical depletion of charge from the nanostructure(s) that originates from (unintentional) modulation doping. In the context of $\mathrm{GaSb} / \mathrm{GaAs}$ heterostructures, acceptor holes will be captured in the deep confining potential of $\mathrm{QD} / \mathrm{QRs}$, which, therefore, will have non-zero hole occupancy even in the absence of photoexcitation. ${ }^{25}$

In this paper, we will discuss the effect of hydrogenation on a GaSb/GaAs sample which previously exhibited OICD. ${ }^{23}$ Post-growth hydrogenation is widely used to engineer the electronic and optical properties of many semiconductors, such as $\mathrm{Si}^{26}{ }^{26} \mathrm{ZnSe},{ }^{27} \mathrm{GaAs},{ }^{28}$ and graphene. ${ }^{29}$ But the effects of hydrogenation are complex: ${ }^{30}$ Hydrogen behaves exclusively as a donor in $\mathrm{ZnO}^{31}$ and as an acceptor in $\mathrm{GaSb}^{32}$ It may also have an amphoteric behaviour, i.e., it can behave as either a donor or acceptor in a given material, depending on the doping being $p$ or $n$ type, respectively. ${ }^{33}$ In GaAs in particular, hydrogen

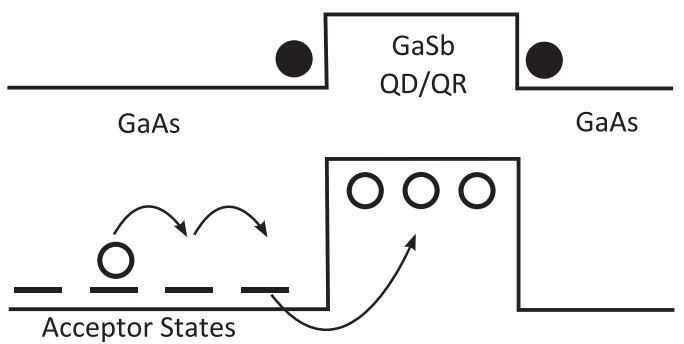

FIG. 1. Band-gap diagram of electrons (solid circles) and holes (hollow circles) in GaSb/GaAs nanostructures. Arrows show the interaction of photogenerated holes with acceptor states before they reach the nanostructure. 
usually passivates the unintentional carbon dopants incorporated during sample growth. ${ }^{34}$ As the vast majority of the material in $\mathrm{GaSb} / \mathrm{GaAs} \mathrm{QD} / \mathrm{QR}$ samples is GaAs, the amphoteric properties of hydrogen are expected to dominate. It is predicted that hydrogenation will suppress or remove OICD by neutralising shallow acceptor states, redshifting the emission. Finally, it should be mentioned that ion bombardment of samples may also introduce shallow defects ${ }^{35}$ or point defects that behave as donors, as in $\mathrm{InN}^{30,36}$

The four hydrogenated samples presented here were taken from a single $\sim 4 \times 4 \mathrm{~mm}$ piece of a $\mathrm{GaSb} / \mathrm{GaAs} \mathrm{QD} /$ QR sample, which was grown by molecular beam epitaxy (MBE) on a $2^{\prime \prime}$ GaAs (100) substrate. ${ }^{8}$ First, a GaAs buffer layer was grown at $580^{\circ} \mathrm{C}$, followed by 2.1 monolayers of $\mathrm{GaSb}$ at $490^{\circ} \mathrm{C}$ with a III/V ratio of 2. An initial 9-nm GaAs cold-cap was grown at $430^{\circ} \mathrm{C}$ with a further $100 \mathrm{~nm}$ of $\mathrm{GaAs}$ grown at $500^{\circ} \mathrm{C}$. Finally, another $\mathrm{GaSb}$ layer was deposited under the same conditions as the first. Atomic force microscopy and cross-sectional transmission electron microscopy showed that the uncapped nanostructures consisted of QDs, but the capped nanostructures consisted of both QDs and QRs. ${ }^{8}$ These images were used to estimate the dimensions of the buried nanostructures: QDs have base lengths of $20 \pm 5 \mathrm{~nm}$ and heights of $5.0 \pm 0.5 \mathrm{~nm}$. The QRs have outer diameters of $30 \pm 7 \mathrm{~nm}$, inner diameters of $10 \pm 3 \mathrm{~nm}$, and ring lobe heights of $4.0 \pm 0.5 \mathrm{~nm}$. The formation of $\mathrm{QRs}$ in capped $\mathrm{GaSb}$ layers is common and results from $\mathrm{Sb}$ migration away from the highly strained QD centre during capping. ${ }^{37,38}$

The sample studied in this paper was previously shown to exhibit OICD in the wetting layer (WL) emission at temperatures, $T,<30 \mathrm{~K}$ and in the QR emission at $T>300 \mathrm{~K}^{23}$ This dependence on temperature was interpreted as the thermalisation of acceptor holes from the WL, where they are located at low temperatures, into the QRs at high temperatures. Highly strained "dark" QD states were used to account for the location of the acceptor holes at intermediate temperatures. It is believed that the majority of the sample's PL emission originates from the less strained QRs, so we shall hereafter refer to the nanostructures as QRs.

The original sample was cleaved into four $\sim 2 \times 2 \mathrm{~mm}$ pieces and hydrogenated using a $100-\mathrm{eV}$ proton beam. The exposure conditions for samples A to D are shown in Table I. The exposure temperatures, $250^{\circ} \mathrm{C}$ and $300^{\circ} \mathrm{C}$, determine the hydrogen penetration depth into the sample, with higher temperatures causing deeper penetration. Both of these temperatures are expected to allow hydrogen to interact with the entire capping layer $(\sim 110 \mathrm{~nm})$ and the GaAs surrounding the GaSb QR layer. These temperatures are also below the

TABLE I. Hydrogenation conditions for the investigated samples.

\begin{tabular}{lccccc}
\hline \hline & \multicolumn{2}{c}{ First exposure } & & \multicolumn{2}{c}{ Second exposure } \\
\cline { 2 - 3 } \cline { 5 - 6 } Sample & $\begin{array}{c}\text { Exposure } \\
\left(\text { atoms } / \mathrm{cm}^{2}\right)\end{array}$ & $\begin{array}{c}\text { Temperature } \\
\left({ }^{\circ} \mathrm{C}\right)\end{array}$ & & $\begin{array}{c}\text { Exposure } \\
\left(\text { atoms } / \mathrm{cm}^{2}\right)\end{array}$ & $\begin{array}{c}\text { Temperature } \\
\left({ }^{\circ} \mathrm{C}\right)\end{array}$ \\
\hline $\mathrm{A}$ & $3 \times 10^{17}$ & 300 & & $\ldots$ & $\ldots$ \\
$\mathrm{B}$ & $1 \times 10^{18}$ & 300 & & $\ldots$ & $\ldots$ \\
$\mathrm{C}$ & $1 \times 10^{17}$ & 300 & & $1 \times 10^{18}$ & 250 \\
$\mathrm{D}$ & $5 \times 10^{16}$ & 300 & & $3 \times 10^{18}$ & 300 \\
\hline \hline
\end{tabular}

$550{ }^{\circ} \mathrm{C}$ temperature required to break the $\mathrm{C}-\mathrm{H}$ pairs and reactivate the carbon acceptors. ${ }^{39}$ Notice that samples C and D have been irradiated a second time at $250^{\circ} \mathrm{C}$ and $300^{\circ} \mathrm{C}$, respectively, to increase acceptor passivation. Finally, from the full width at half maximum of the acceptor band, typically $\sim 10 \mathrm{meV}$, it is possible to estimate an upper limit of the carbon impurity concentration as of the order of $10^{18} \mathrm{~cm}^{-3}$. ${ }^{39}$ It follows that the areal density of C impurities in the $\sim 100 \mathrm{~nm}$ GaAs capping layer can be estimated to be of the order of $10^{13} \mathrm{~cm}^{-2}$. This is significantly smaller than the minimum areal density of the irradiated hydrogen atoms, which, if a $99 \%$ reflection for $100 \mathrm{eV}$ protons is assumed, is estimated to be $\sim 3 \times 10^{15} \mathrm{~cm}^{-2}$ in the case of sample A. Additionally, for carbon concentrations lower than $10^{19} \mathrm{~cm}^{-3}$, the $\mathrm{C}_{\mathrm{As}}-\mathrm{H}$ complex is expected to be the dominant species involving $\mathrm{C}$ and $\mathrm{H}^{40}$ Therefore, hydrogenation is expected to passivate acceptor states in the samples.

PL measurements were made using a 532-nm laser and a Peltier-cooled InGaAs array detector. Laser light was delivered using a $200-\mu \mathrm{m}$-core optical fibre, which illuminated $\sim 2 \mathrm{~mm}^{2}$ of the sample surface. PL emission was collected by a $550-\mu \mathrm{m}$-core optical fibre for measurement by a spectrometer and the detector. For $4.2 \mathrm{~K}$ measurements, the sample was immersed in liquid helium, while a continuousflow Oxford Instruments cryostat was used for measurements at $\geq 300 \mathrm{~K}$.

We start with the $4.2 \mathrm{~K}$ data, which is shown in Fig. 2. The amount of hydrogen each sample was exposed to goes
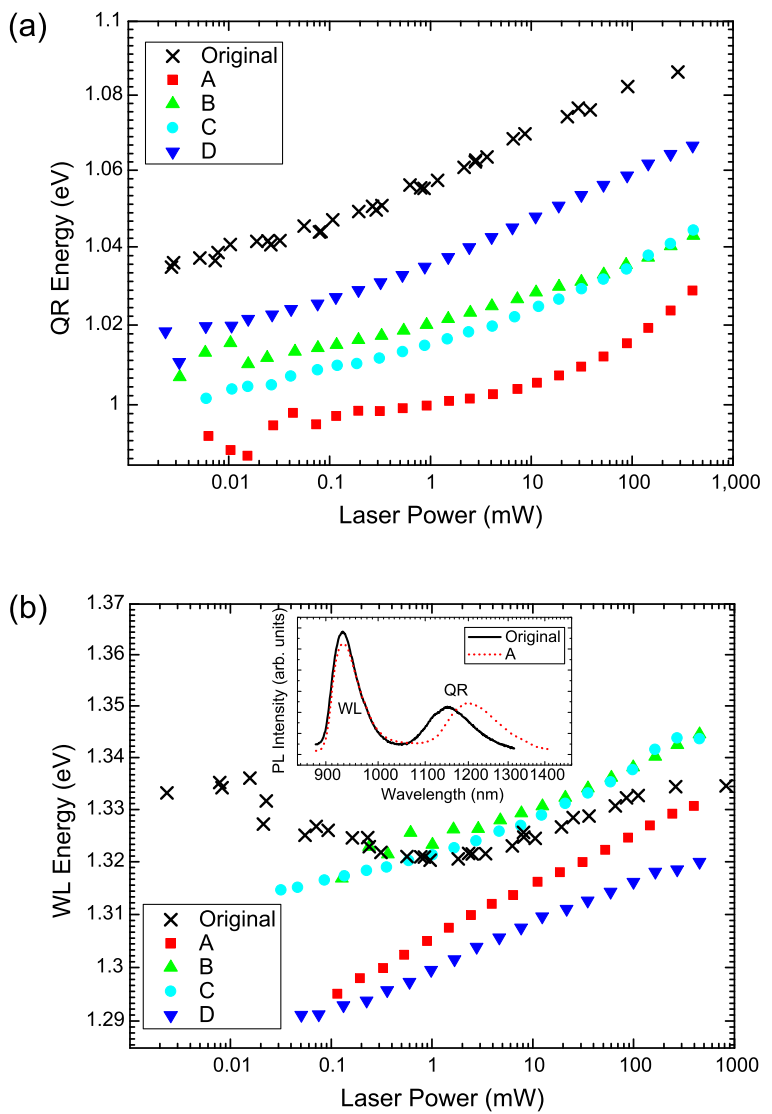

FIG. 2. Emission energies at $4.2 \mathrm{~K}$ for (a) the QR and (b) the WL as a function of excitation power. Data are shown for the original sample (measured at $2 \mathrm{~K}$ ) and the four hydrogenated pieces. The inset shows high power PL spectra of the original sample and sample A. 
in ascending order from samples A to D. Assuming that the greater the hydrogen exposure, the more acceptor states are passivated, then any changes due to hydrogenation should increase from sample A (smallest change) to sample D (greatest change). This would lead to an inverse relationship between hydrogen exposure and emission energy, as hydrogen passivates acceptor states and hence reduces charging of the nanostructures. By the same reasoning, the effect of OICD would be expected to be reduced with increasing hydrogen exposure.

It can be seen in Fig. 2 that these predictions are partly realised. The QR emission [Fig. 2(a)] does show a reduction in energy as a result of hydrogenation, but the magnitude of the reduction does not show the expected dependence on hydrogen dose: Sample A has the greatest reduction and sample D the smallest reduction, while samples B and C are almost indistinguishable. This relationship is the opposite of that expected and will be discussed later in light of the high temperature data. The reduction of emission energy in the WL [Fig. 2(b)] is less clear and there appears to be no correlation between PL energy and hydrogen dose. For the WL, this may be expected, since, unlike QRs, it does not experience capacitive charging: Any change in the WL hole occupancy should have a much less dramatic effect on the PL energy. Overall, there is no clear correlation between absolute emission energy and hydrogen exposure for either the QRs or the WL.

Nevertheless, hydrogenation has clearly had a striking effect on the power-varying data for the WL. Fig. 2(b) shows that all of the hydrogenated samples display a monotonic blueshift with increasing laser power, in contrast to the original sample which displayed a significant redshift at low laser powers due to OICD. Since the OICD redshift involves the interaction of the nanostructure with dopants, this result suggests that hydrogenation has passivated the background carbon acceptors which were active in the original sample. This is consistent with the level of hydrogen dose applied to all samples.

We now go on to discuss the high-temperature data. In the original sample, OICD was seen in the QR powervarying data at temperatures above $300 \mathrm{~K}$. An increase in the magnitude of OICD with increasing temperature was attributed to the thermally induced migration of acceptor holes into the QRs. In stark contrast, it can be seen in Fig. 3(a) that OICD is not observed in any of the hydrogenated samples at $360 \mathrm{~K}$. Also, as was seen at $4.2 \mathrm{~K}$, the QR emission energy has been reduced as a result of hydrogenation. However, there is now no clear difference between the hydrogenated samples, with all four showing a $\sim 30 \mathrm{meV}$ redshift relative to the original sample. It was previously shown in a similar $\mathrm{GaSb} / \mathrm{GaAs}$ QR sample that the capacitive charging energy was $24 \mathrm{meV}$ per additional hole in the QR. ${ }^{21}$ Therefore, at high excitation powers, the reduction in $\mathrm{QR}$ emission energy of $\sim 30 \mathrm{meV}$ after hydrogenation corresponds to a decrease in the average QR occupancy of approximately 1 hole.

The convergence to a common emission energy at high temperature may indicate thermalisation of carriers to an equilibrium state, where charge is evenly spread amongst all QRs in the ensemble. We propose that small dissimilarities in the homogeneity of the individual hydrogenated samples
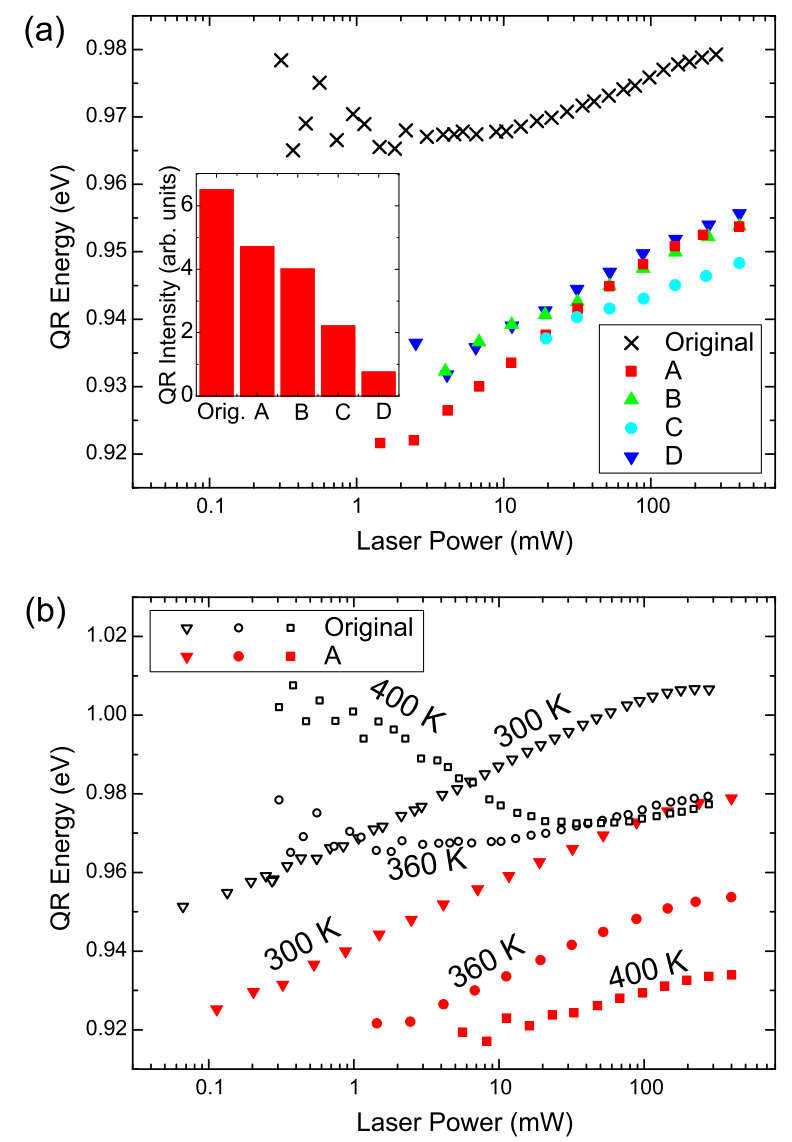

FIG. 3. High temperature power-varying QR data showing: (a) the QR emission energies for all samples at $360 \mathrm{~K}$. The inset shows the emission intensity at the highest excitation power and $300 \mathrm{~K}$ for all samples. (b) A comparison of sample A with the original sample at three different temperatures.

can lead to differences in the photo-induced charging of nanostructures at low temperatures. Indeed, fluctuations in the WL valence band edge were used to explain the localisation of carriers in the WL of this sample at temperatures below $20 \mathrm{~K}^{23}$ and have also been used to explain similar behaviour in other systems. ${ }^{41,42}$ This explains the range of emission energies seen in the $4.2 \mathrm{~K}$ data of the hydrogenated samples and, more specifically, indicates that the inverse relationship between change in emission energy and hydrogen dose seen for samples A and D in Fig. 2(a) is possibly coincidental.

The emission intensity of all four hydrogenated samples was lower than the original sample [Fig. 3(a), inset], which made high-temperature, low-laser-power measurements challenging. Since the recombination dynamics and electron binding in this type-II system are dominated by Coulomb forces, this observation is consistent with a reduction in QR charging due to acceptor passivation. However, quenching of the emission intensity can also be caused by the creation of defects states in the sample during hydrogenation. Evidence for this is provided by the inset to Fig. 3(a), which shows a systematic decrease in intensity with increasing hydrogen dose.

Sample A, with the lowest hydrogen dose, has the highest emission intensity of all of the hydrogenated samples. For this reason, it was decided to investigate this sample further, as emission can be observed at the lowest excitation 
powers and highest temperatures. Since carriers are expected to increasingly migrate into the QRs at higher temperatures and the effect of OICD is greatest at lower excitation powers, sample A provides the best opportunity to observe OICD. In addition, if any sample had retained the active acceptor states that lead to OICD, it should be sample A, because it had the lowest hydrogen exposure. The high-temperature power-varying data of sample $\mathrm{A}$ are compared to the original sample in Fig. 3(b). The original sample shows a striking redshift with increasing excitation (OICD) at $T>300 \mathrm{~K}$, but no OICD is present for the hydrogenated sample. This supports our thesis that hydrogenation has passivated the carbon acceptors responsible for the unintentional doping of $\mathrm{GaSb}$ nanostructures. If this is the case, then the $30-\mathrm{meV}$ redshift in the emission from the hydrogenated samples compared to the original in Fig. 3(a) seems modest. In Ref. 25, it was found that the peak in the (low temperature) PL at very low excitation powers corresponded to a QR occupation of 4 acceptor holes. Indeed, for a capacitive charging energy of $24 \mathrm{meV}, 4$ holes would raise the hole ground state energy by $96 \mathrm{meV}$, taking the projected emission energy for a typical GaSb/GaAs QD/QR from $0.81 \mathrm{eV}$ to $0.906 \mathrm{eV}(1368 \mathrm{~nm})$, which is very similar to what is observed in experiments. We suggest that multiple occupancy of $\mathrm{GaSb} / \mathrm{GaAs} \mathrm{QD} / \mathrm{QRs}$ may be required to generate a sufficiently attractive Coulomb potential to bind electrons in an excitonic state and generate intense emission, i.e., room temperature PL may only be observable at occupancies of a few holes, irrespective of whether they are from background doping or photo-excitation. Indeed, it is quite interesting to note that in Fig. 3(b) the PL of sample A consistently quenches at $\sim 0.92 \mathrm{eV}(1350 \mathrm{~nm})$ irrespective of the temperature. The implication of this is that it may be very difficult to achieve high intensity long wavelength emission $(1550 \mathrm{~nm})$ in this system.

In summary, we have shown that hydrogenation of a $\mathrm{GaSb} / \mathrm{GaAs} \mathrm{QR}$ sample has removed OICD from both the WL and QRs. Our results indicate that even the lowest hydrogen exposure $\left(10^{17}\right.$ atoms $/ \mathrm{cm}^{2}$ at $\left.300{ }^{\circ} \mathrm{C}\right)$ neutralised the carbon acceptor states which were active in the OICD process. Given that the (low-temperature) peak in PL emission for similar (unhydrogenated) samples has previously been shown to correspond to an occupancy of 4 acceptor holes, ${ }^{25}$ the expected redshift in PL emission at $>300 \mathrm{~K}$ due to removal of acceptor holes was found to be very modest; roughly equivalent to just 1 hole. We conclude that multiplehole occupation of $\mathrm{QD} / \mathrm{QRs}$ may be required for intense emission, irrespective of whether they originate from background doping or photo-excitation. Hence, the extension of room-temperature emission of $\mathrm{GaSb} / \mathrm{GaAs} \mathrm{QD} / \mathrm{QRs}$ further into the telecoms band will be challenging.

This work was supported by the EPSRC in the framework of the QD2D project [Grant No. EP/H006419] and QinetiQ (Agreement No: 3000127730).

${ }^{1}$ D. Bimberg, M. Grundmann, and N. Ledentsov, Quantum Dot Heterostructures (Wiley, New York, 1999).

${ }^{2}$ T. Y. Gu, M. A. El-Emawy, K. Yang, A. Stintz, and L. F. Lester, Appl. Phys. Lett. 95, 261106 (2009).
${ }^{3}$ A. J. Shields, Nat. Photonics 1, 215 (2007).

${ }^{4}$ P. J. Carrington, A. S. Mahajumi, M. C. Wagener, J. R. Botha, Q. Zhuang, and A. Krier, Physica B 407, 1493 (2012).

${ }^{5}$ B. Bansal, M. Hayne, M. Geller, D. Bimberg, and V. V. Moshchalkov, Phys. Rev. B 77, 241304(R) (2008).

${ }^{6}$ M. Geller, A. Marent, T. Nowozin, D. Bimberg, N. Akcay, and N. Oncan, Appl. Phys. Lett. 92, 092108 (2008).

${ }^{7}$ K. Gradkowski, T. J. Ochalski, N. Pavarelli, H. Y. Liu, J. Tatebayashi, D. P. Williams, D. J. Mowbray, G. Huyet, and D. L. Huffaker, Phys. Rev. B 85, 035432 (2012).

${ }^{8}$ M. Ahmad Kamarudin, M. Hayne, R. J. Young, Q. D. Zhuang, T. Ben, and S. I. Molina, Phys. Rev. B 83, 115311 (2011).

${ }^{9}$ R. B. Laghumavarapu, A. Moscho, A. Khoshakhlagh, M. El-Emawy, L. F. Lester, and D. L. Huffaker, Appl. Phys. Lett. 90, 173125 (2007).

${ }^{10}$ A. Marent, T. Nowozin, M. Geller, and D. Bimberg, Semicond. Sci. Technol. 26, 014026 (2011).

${ }^{11}$ M. Hayne, R. J. Young, E. P. Smakman, T. Nowozin, P. Hodgson, J. K. Garleff, P. Rambabu, P. M. Koenraad, A. Marent, L. Bonato, A. Schliwa, and D. Bimberg, J. Phys. D: Appl. Phys. 46, 264001 (2013).

${ }^{12}$ J. Tatebayashi, A. Khoshakhlagh, S. H. Huang, G. Balakrishnan, L. R. Dawson, and D. L. Huffaker, Appl. Phys. Lett. 90, 261115 (2007).

${ }^{13}$ T. H. Loeber, D. Hoffmann, and H. Fouckhardt, Beilstein J. Nanotechnol. 2, 333 (2011).

${ }^{14}$ W. H. Lin, K. W. Wang, S. Y. Lin, and M. C. Wu, IEEE Photonics Technol. Lett. 25, 97 (2013).

${ }^{15}$ T. Nowozin, A. Marent, L. Bonato, A. Schliwa, D. Bimberg, E. P. Smakman, J. K. Garleff, P. M. Koenraad, R. J. Young, and M. Hayne, Phys. Rev. B 86, 035305 (2012).

${ }^{16}$ T. Nowozin, L. Bonato, A. Hogner, A. Wiengarten, D. Bimberg, W.-H. Lin, S.-Y. Lin, C. J. Reyner, B. L. Liang, and D. L. Huffaker, Appl. Phys. Lett. 102, 052115 (2013).

${ }^{17}$ F. Hatami, M. Grundmann, N. N. Ledentsov, F. Heinrichsdorff, R. Heitz, J. Bohrer, D. Bimberg, S. S. Ruvimov, P. Werner, V. M. Ustinov, P. S. Kop'ev, and Z. I. Alferov, Phys. Rev. B 57, 4635 (1998).

${ }^{18}$ Y. S. Chiu, M. H. Ya, W. S. Su, and Y. F. Chen, J. Appl. Phys. 92, 5810 (2002).

${ }^{19}$ L. Muller-Kirsch, R. Heitz, A. Schliwa, O. Stier, D. Bimberg, H. Kirmes, and W. Neumann, Appl. Phys. Lett. 78, 3908 (2001).

${ }^{20}$ D. Alonso-Alvarez, B. Alen, J. M. Garcia, and J. M. Ripalda, Appl. Phys. Lett. 91, 263103 (2007).

${ }^{21}$ P. D. Hodgson, R. J. Young, M. A. Kamarudin, P. J. Carrington, A. Krier, Q. D. Zhuang, E. P. Smakman, P. M. Koenraad, and M. Hayne, J. Appl. Phys. 114, 073519 (2013).

${ }^{22}$ M. Hayne, O. Razinkova, S. Bersier, R. Heitz, L. Muller-Kirsch, M. Geller, D. Bimberg, and V. V. Moshchalkov, Phys. Rev. B 70, 081302(R) (2004).

${ }^{23}$ P. D. Hodgson, R. J. Young, M. A. Kamarudin, Q. D. Zhuang, and M. Hayne, Phys. Rev. B 88, 155322 (2013).

${ }^{24}$ M. Hayne, A. Usher, A. S. Plaut, and K. Ploog, Phys. Rev. B 50, 17208 (1994).

${ }^{25}$ R. J. Young, E. P. Smakman, A. M. Sanchez, P. Hodgson, P. M. Koenraad, and M. Hayne, Appl. Phys. Lett. 100, 082104 (2012).

${ }^{26}$ Y. Qiu, O. Kunz, A. Fejfar, M. Ledinsky, B. T. Chan, I. Gordon, D. Van Gestel, S. Venkatachalm, and R. Egan, Sol. Energy Mater. Sol. Cells 122, 31 (2014).

${ }^{27}$ M. D. Kim, H. S. Park, and T. W. Kim, J. Appl. Phys. 84, 3125 (1998).

${ }^{28}$ G. D. Azevedo, J. H. D. da Silva, and E. Avendano, Nucl. Instrum. Methods Phys. Res., Sect. B 238, 329 (2005).

${ }^{29}$ D. C. Elias, R. R. Nair, T. M. G. Mohiuddin, S. V. Morozov, P. Blake, M. P. Halsall, A. C. Ferrari, D. W. Boukhvalov, M. I. Katsnelson, A. K. Geim, and K. S. Novoselov, Science 323, 610 (2009).

${ }^{30}$ Hydrogen in Semiconductors, edited by J. I. Pankove and N. M. Johnsom (Academic Press, 1991).

${ }^{31}$ C. G. Van de Walle, Phys. Rev. Lett. 85, 1012 (2000).

${ }^{32}$ C. G. Van de Walle and J. Neugebauer, Nature 423, 626 (2003).

${ }^{33}$ C. G. Van de Walle and J. Neugebauer, in Annual Review of Materials Research (Annual Reviews, Palo Alto, 2006), Vol. 36, p. 179.

${ }^{34}$ A. A. Bonapasta, Phys. Rev. B 48, 8771 (1993).

${ }^{35}$ S. X. Li, K. M. Yu, J. Wu, R. E. Jones, W. Walukiewicz, J. W. Ager, W. Shan, E. E. Haller, H. Lu, and W. J. Schaff, Phys. Rev. B 71, 161201 (2005).

${ }^{36}$ G. Pettinari, F. Masia, M. Capizzi, A. Polimeni, M. Losurdo, G. Bruno, T. H. Kim, S. Choi, A. Brown, V. Lebedev, V. Cimalla, and O. Ambacher, Phys. Rev. B 77, 125207 (2008). 
${ }^{37}$ E. P. Smakman, J. K. Garleff, R. J. Young, M. Hayne, P. Rambabu, and P. M. Koenraad, Appl. Phys. Lett. 100, 142116 (2012).

${ }^{38}$ A. J. Martin, J. Hwang, E. A. Marquis, E. Smakman, T. W. Saucer, G. V. Rodriguez, A. H. Hunter, V. Sih, P. M. Koenraad, J. D. Phillips, and J. Millunchick, Appl. Phys. Lett. 102, 113103 (2013).

${ }^{39}$ D. M. Kozuch, M. Stavola, S. J. Pearton, C. R. Abernathy, and W. S. Hobson, J. Appl. Phys. 73, 3716 (1993).
${ }^{40}$ D. M. Kozuch, M. Stavola, S. J. Pearton, C. R. Abernathy, and J. Lopata, Appl. Phys. Lett. 57, 2561 (1990).

${ }^{41}$ L. Brusaferri, S. Sanguinetti, E. Grilli, M. Guzzi, A. Bignazzi, F. Bogani, L. Carraresi, M. Colocci, A. Bosacchi, P. Frigeri, and S. Franchi, Appl. Phys. Lett. 69, 3354 (1996).

${ }^{42}$ T. Nuytten, M. Hayne, M. Henini, and V. V. Moshchalkov, Phys. Rev. B 77, 115348 (2008). 